PSICOLOGÍA

IBEROAMERICANA
Psicología Iberoamericana ISSN: 1405-0943

revista.psicologia@ibero.mx

Universidad Iberoamericana, Ciudad de México México

\title{
Adaptación y validación de la escala de tecnoadicción del cuestionario red- tecnoestrés, en una población laboral mexicana
}

Villavicencio-Ayub, Erika; Cazares Vargas, Maira Alicia

Adaptación y validación de la escala de tecnoadicción del cuestionario red-tecnoestrés, en una población laboral mexicana

Psicología Iberoamericana, vol. 29, núm. 1, 2021

Universidad Iberoamericana, Ciudad de México, México

Disponible en: https://www.redalyc.org/articulo.oa?id=133967526002

DOI: https://doi.org/10.48102/pi.v29i1.176

Los autores/as conservan los derechos de autor y ceden a la revista el derecho de la primera publicación, con la obra registrada bajo la Licencia Creative Commons Atribución 4.0 Internacional.

Los autores/as conservan los derechos de autor y ceden a la revista el derecho de la primera publicación, con la obra registrada bajo la Licencia Creative Commons Atribución 4.0 Internacional.

\section{(c) (7)}

Esta obra está bajo una Licencia Creative Commons Atribución 4.0 Internacional. 
Artículos

\title{
Adaptación y validación de la escala de tecnoadicción del cuestionario red- tecnoestrés, en una población laboral
} mexicana

\author{
Adaptation and validation of the Techno Addiction Scale in \\ the Red-Technostress Questionnaire, in a Mexican labour \\ population \\ Erika Villavicencio-Ayub erikavilla@hotmail.com \\ Universidad Nacional Autónoma de México, México \\ (1) https://orcid.org/0000-0003-0021-5020 \\ Maira Alicia Cazares Vargas alicia.cazares7x@gmail.com \\ Universidad Nacional Autónoma de México, México \\ https://orcid.org/0000-0003-0544-7058
}

Psicología Iberoamericana, vol. 29, núm. 1,2021

Universidad Iberoamericana, Ciudad de México, México

Recepción: 14 Julio 2020

Aprobación: 11 Noviembre 2020

DOI: https://doi.org/10.48102/ pi.v29i1.176

Redalyc: https://www.redalyc.org/ articulo.oa?id=133967526002
Resumen: La tecnoadicción aparece cuando una persona genera una dependencia psicológica hacia un dispositivo tecnológico, lo que implica un uso excesivo y compulsivo. El propósito de este estudio, fue adaptar y validar la Escala de Tecnoadicción incluida en el Cuestionario RED-Tecnoestrés de Salanova et al. (2013), en una población de 1,270 trabajadores mexicanos de edades entre los 18 y 69 años, donde el 62.39\% fueron mujeres. El estudio fue de tipo cuantitativo no experimental, ex post facto. Los datos fueron obtenidos mediante un muestreo no probabilístico por conveniencia. La estructura factorial resultante del análisis factorial exploratorio (varimax), quedó compuesta por dos factores, que explican el $60.79 \%$ de la varianza. El análisis factorial confirmatorio corroboró el modelo teórico de Llorens et al. (2011), que incluye el uso excesivo de la tecnología, compuesto por tres reactivos y uso compulsivo de la tecnología, compuesto por cinco reactivos con un buen ajuste $(\alpha=.845$, NFI=.965, $\mathrm{TLI}=.958, \mathrm{CFI}=.971$ y RMSEA=.066) lo que sugiere que el instrumento es apto para medir tecnoadicción en población mexicana.

Palabras clave: tecnoadicción, adicción digital, adicción a TIC, dependencia tecnológica, desorden de adicción a internet, ciberadicción.

Abstract: Techno-addiction occurs when someone generates a psychological dependence on technological devices, characterized by excessive and compulsive use of these. This study's purpose was to adapt the Salanova et al. (2013) Techno Addiction Scale in a population of 1270 Mexican employees, where $62.39 \%$ were women and between the ages of 15 and 69-years-old. The study was ex-post facto. The resultant factorial structure of the exploratory factor analysis (varimax rotation) was integrated by two factors that explain $60.79 \%$ of the variance. The scale confirmatory factor analysis confirmed the theoretical model of Llorens et al. (2011), excessive use of technology, integrated by three items, and compulsive use of technology, integrated by five items, with a good fit $(\alpha=.845$, NFI=.965, TLI=.958, CFI=.971 y RMSEA =.066). We conclude that the scale has psychometric properties necessary for the Mexican population.

Keywords: techno addiction, digital addiction, ICT addiction, technology dependence, internet addiction disorder, cyber addiction. 


\section{Introducción}

La adicción a las Tecnologías de la Información y la Comunicación (TIC), es un tipo de adicción conductual que aparece cuando una persona genera una dependencia psicológica hacia un dispositivo tecnológico (internet, redes sociales, celulares, aplicaciones o videojuegos), lo que conlleva a repercusiones negativas en su salud, vida social, familia y trabajo (Sánchez-Carbonell, 2014).

Llorens y colaboradores (2011), se refieren a este fenómeno con el término tecnoadicción y lo explican mediante un modelo de dos factores: el uso excesivo y el uso compulsivo de la tecnología. El primero, se refiere a que los usuarios tienden a asignar una excepcional cantidad de tiempo al uso de las TIC, mientras que el segundo factor, explica que estas personas con frecuencia y persistencia piensan en las TIC (incluso cuando no están usándolas), lo que sugiere que puedan sentirse obsesionados con ellas. A través de dicho comportamiento, los usuarios buscan obtener un nivel adecuado de satisfacción que les permita evitar el malestar generado por la no utilización de tecnologías, algo relativamente similar a un síndrome de abstinencia caracterizado por un profundo malestar emocional que puede manifestarse con mal humor, estado de ánimo disfórico, insomnio, irritabilidad e inquietud psicomotora (Cía, 2013; Gil, 2015).

Haciendo una analogía, uno de los factores que determinan la capacidad de generar adicción a una sustancia, son sus capacidades reforzadoras. Se acepta que cuando más intenso sea el refuerzo (efecto) y menos tiempo transcurra entre el consumo y la respuesta, más capacidad tiene la sustancia de generar dependencia (Sánchez-Carbonell, 2014). En el caso de las TIC, pasaría lo mismo, ya que cuentan con ciertas características que promueven la aparición de conductas adictivas, como lo son:

La facilidad de acceso, rapidez e inmediatez en la conexión, funcionamiento las veinticuatro horas del día, posibilidad de acceder a todo tipo de contenidos y estímulos, facilitación de las relaciones sociales, recompensa en un corto periodo de tiempo, desafío y nivel creciente de dificultad junto con la sensación de control o dominio en los juegos, visibilidad y reafirmación de la identidad en el grupo, conexión permanente con los amigos, alivio de las tensiones y del malestar emocional, desconexión de la realidad, posibilidad de crear personalidades ficticias, anonimato, etcétera (Terán Prieto, 2019, p. 136).

Existen varios instrumentos para medir la adicción a las TIC, como el Cuestionario de Adicción a Internet y a los Videojuegos para Adolescentes de Chahín y Libia (2016), la Escala de Adicción a Videojuegos para Adolescentes (GASA) de Lloret et al. (2017), SPAI: Smartphone Addiction Inventory de Lin et al. (2014), y la Escala de adicción a las redes sociales de Cabero-Almenara et al. (2020), por mencionar algunos. Todos tienen en común que evalúan la adicción a las TIC de forma aislada, concentrándose sólo en un tipo de dispositivo tecnológico. Es por ello que dentro de la literatura, destaca la Escala de Tecnoadicción incluida en el Cuestionario RED-Tecnoestrés de Salanova 
y colaboradores (2013), la cual considera a todas las TIC (véanse los reactivos de la escala original en la Tabla 1 ).

Tabla 1

Tabla 1

Reactivos de la escala original, agrupados por factor

\begin{tabular}{|c|c|c|}
\hline Factor & Reactivos & $\begin{array}{l}\text { Consistencia } \\
\text { interna (alfa } \\
\text { de Cronbach) } \\
\text { de la escala. }\end{array}$ \\
\hline \multirow{3}{*}{$\begin{array}{l}\text { Uso excesivo de la } \\
\text { Tecnología }\end{array}$} & $\begin{array}{l}\text { Creo que utilizo en exceso las tecnologías en } \\
\text { mi vida. }\end{array}$ & \multirow{6}{*}{0.85} \\
\hline & $\begin{array}{l}\text { Utilizo continuamente las tecnologías, incluso } \\
\text { fuera de mi horario de trabajo. }\end{array}$ & \\
\hline & $\begin{array}{l}\text { Dedico más tiempo a las tecnologías que a } \\
\text { estar con amigos y familiares o a practicar } \\
\text { hobbies o actividades de tiempo libre. }\end{array}$ & \\
\hline \multirow{3}{*}{$\begin{array}{l}\text { Uso Compulsivo de la } \\
\text { Tecnología }\end{array}$} & $\begin{array}{l}\text { Me encuentro pensando en tecnologías } \\
\text { continuamente (por ejemplo, revisar el correo } \\
\text { electrónico, búsqueda de información en } \\
\text { internet, etc) incluso fuera del horario de } \\
\text { trabajo. }\end{array}$ & \\
\hline & $\begin{array}{l}\text { Me siento mal si no tengo acceso a las } \\
\text { tecnologías (Internet, correo electrónico, móvil, } \\
\text { etc). }\end{array}$ & \\
\hline & $\begin{array}{l}\text { Siento que un impulso interno me obliga a } \\
\text { utilizar las tecnologías en cualquier momento y } \\
\text { lugar. }\end{array}$ & \\
\hline
\end{tabular}

Fuente: Salanova et al. (2013, p. 426).

Salanova et al. (2013, p. 426).

En lo reportado, encontramos análisis del papel del género en la incidencia de la tecnoadicción, siendo los hombres quienes más presentan el fenómeno de la adicción al internet (Aznar-Díaz et al., 2020; Kircaburun \& Griffiths, 2018; Li et al., 2014; Ruiz-Palmero et al., 2019). Otras investigaciones reportan la incidencia del género de acuerdo con el tipo de TIC como, por ejemplo, los varones suelen experimentar más adicción a los videojuegos que las mujeres (Gentile et al., 2011), y mientras los hombres pasan más tiempo en las videoconsolas, las mujeres pasan más tiempo en redes sociales y chat (Vink et al., 2015).

En cuanto a las consecuencias de la tecnoadicción, estudios reportan que la adicción al internet puede provocar aislamiento, irascibilidad, abandono de actividades y descuido de la higiene personal (Chen et al., 2004; Tsitsika et al., 2013). Otros autores han encontrado que esta experiencia puede provocar fatiga, cansancio mental, ansiedad (Salanova et al., 2013), depresión e ideación suicida (Garcia-Priego et al., 2020; Jasso-Medrano \& Lopez-Rosales, 2018), trastornos de la alimentación (Kim et al., 2010), distrés (McEwen, 2006; Riedl, 2012; Shapira et al., 2003), procrastinación (Aznar-Díaz et al., 2020), problemas económicos derivados del gasto financiero que dedica el tecnoadicto para estar al último grito de la moda y reducción del rendimiento y desempeño en el puesto de trabajo (Sánchez-Carbonell et al., 2008). 
Principalmente las investigaciones sobre tecnoadicción se concentran en Asia, Estados Unidos y Europa, no obstante -en el caso de México- la mayoría de los estudios reportados muestran la relación de estas variables en el sector académico y se focalizan en un solo tipo de tecnoadicción ya sea la adicción al internet, redes sociales o Smartphone (Aznar-Díaz et al., 2020; Bernal Nava, 2011; Contreras et al., 2019; Garcia-Priego et al., 2020; Jasso-Medrano \& Lopez-Rosales, 2018). Cabe destacar, que en la revisión de la literatura encontramos sólo dos estudios de tipo psicométrico, en donde se adaptan y validan las siguientes escalas: Social Media Addiction Scale-Student Form (SMAS-SF) de Cabero-Almenara et al. (2020), y el Internet Addiction Test (IAT) de Kimberley Young (Hilt et al., 2015).

Además de los estudios antes mencionados, México cuenta con múltiples estadísticas que ofrecen una aproximación al entendimiento de la variable que aquí estudiamos. De acuerdo con los resultados de la Encuesta Nacional Sobre Disponibilidad y Uso de Tecnologías de la Información en los Hogares (Instituto Nacional de Estadística y Geografía [INEGI], Secretaría de Comunicaciones y Transportes, Instituto Federal de Telecomunicaciones [IFT], 2020), en México existen 80.6 millones de internautas ( $70.1 \%$ de la población de seis años o más) y 20.1 millones de hogares disponen de Internet (56.4\%), ya sea mediante conexión fija o móvil. Cabe resaltar que entre los años 2017 y 2019 hubo un incremento de internautas en zonas urbanas (de $71.2 \%$ a $76.6 \%$ ) y rurales (de $39.2 \%$ a $47.7 \%$ ).

En este estudio, los usuarios de las TIC han demostrado mayor preferencia por los celulares inteligentes (Smartphone), que por las computadoras; se estima que en el país existen 86.5 millones de usuarios de esta tecnología (75.1\% de la población de seis años o más) y 49.4 millones de usuarios de computadora ( $43.0 \%$ de la población de seis años o más). Por otro lado, un estudio sobre los hábitos de los usuarios de internet revela que el $67 \%$ de los internautas mexicanos, perciben que se encuentran conectados las veinticuatro horas del día; no obstante, el tiempo promedio de conexión a internet en México, es de 8 horas con 20 minutos, 15 minutos más que lo reportado en 2018 (Asociación de Internet MX, 2019).

Tras la reforma de telecomunicaciones en 2013, México ha implementado diversos programas de inclusión digital que permiten el acceso a la información para toda la población (Organización para la Cooperación y el Desarrollo Económicos [OCDE], 2017). Proyectos como México Conectado, instalación de internet de banda ancha y bases eléctricas para cargar dispositivos en vía pública (por mencionar algunos) fomentan en la población, el uso excesivo de las tecnologías. Lo anterior, podría dar pauta a comportamientos asociados a la variable tecnoadicción, ya que actualmente no existe, una campaña de prevención que oriente en el uso eficiente y saludable de las TIC.

No debe pasarse por alto que las adicciones a las TIC, aún no se encuentran tipificadas como trastorno, pero vale la pena mencionar que en el DSM-V-Manual Diagnóstico y Estadístico de los Trastornos 
Mentales (American Psychiatric Association, 2014), en la categoría denominada 'Trastornos adictivos y relacionados a sustancias', ya son tomados en cuenta los 'Trastornos no relacionados a sustancias', como lo son el 'Trastorno por Juego de Apuestas y el Trastorno por Juegos de Internet', que aún requiere mayor investigación (Cía, 2013; Simó Sanz et al., 2017; Terán Prieto, 2019). Estudios como el que se reporta a continuación, contribuyen a recabar mayor evidencia en este fenómeno con instrumentos que reúnen las propiedades psicométricas necesarias para su utilización en la población nacional.

\section{Objetivo}

El objetivo del presente estudio consistió en adaptar y validar la Escala de Tecnoadicción, que se encuentra incluida en el cuestionario REDTecnoestrés de Salanova et al. (2013), en una población de trabajadores mexicanos.

\section{Método}

\section{Procedimiento}

Estuvo dividido en dos fases. La primera, incluyó la adaptación cultural de la escala. Se inició con la modificación de reactivos vigilando la equivalencia cultural y en el lenguaje, se redactaron nuevos reactivos para tener suficientes en caso de que los originales fueran eliminados durante el tratamiento psicométrico.

La evaluación de la validez de contenido se realizó mediante evaluación de interjueces, en donde cada juez calificó y asentó en una tabla la siguiente calificación: 1 punto significaba que estaban de acuerdo con el reactivo en su totalidad, 0.5 que requería algunos cambios en redacción y/o palabras, y 0 si el reactivo se debía eliminar.

Con ello se efectuó la aplicación de una prueba piloto, la evaluación de la aplicabilidad del análisis factorial exploratorio mediante $\mathrm{KMO}$ (teniendo como criterio de puntaje el rango 0.5 a 1 ) y el test de esfericidad de Bartlett (considerando como válidos aquellos resultados cuya fiabilidad fuera menor a 0.05 ) y el análisis factorial exploratorio a fin de obtener la validez de constructo, en donde se consideraron aquellos reactivos agrupados a un factor cuyos coeficientes fueran mayores o iguales a 0.4 (Hernández Sampieri et al., 2014; Montoya, 2007; Reyes \& Barragán, 2008).

La segunda fase, consistió en evaluar las estructuras factoriales resultantes del análisis factorial exploratorio, mediante la técnica de ecuaciones estructurales en AMOS (Escobedo Portillo et al., 2016), a fin de confirmar el modelo de 2 factores propuesto por Llorens et al. (2011). Para evaluar la calidad del modelo, verificamos que los estadísticos de bondad de ajuste cumplieran con los siguientes criterios: 1) Chi-cuadrada mayor a 0.05 (no significativo), en caso de ser menor, el índice CMIN/ 
DF debe ser menor a tres; 2) CFI, TLI y NFI mayores o iguales a 0.95; y 3) RMSEA menor a 0.08 (Ruiz et al., 2010). Además, se verificó que las cargas factoriales del modelo fueran $\geq$ a 0.07 , lo que, de acuerdo con Escobedo Portillo et al. (2016) señala la correlación existente entre las variables y los factores. Por último, evaluamos la confiabilidad mediante la medida de consistencia interna alfa de Cronbach considerando el rango 0.70 a 0.90 como aceptables (Hernández Sampieri et al., 2014).

\section{Muestra}

Para ambas fases, el muestreo fue no probabilístico por conveniencia, con consentimiento informado y anonimato. Participaron trabajadores de organizaciones ubicadas en la Ciudad de México y en el Estado de México, el $36.5 \%$ laboraba en instituciones públicas y el $63.5 \%$ en privadas, todos ellos mayores de edad. Para ello, se enviaron invitaciones a diferentes organizaciones con quienes previamente habíamos colaborado en otros proyectos, se explicó el objetivo del estudio y se ofrecieron los resultados de sus empleados, incluyendo recomendaciones para realizar programas de desarrollo y capacitación. Una vez que obtuvimos las confirmaciones de participación, acudimos con el encargado del área de recursos humanos para coordinar las fechas de aplicación y el comunicado donde se plasmaba la información del proyecto.

En la prueba piloto de la fase 1, participaron 289 trabajadores mexicanos, $47.8 \%$ eran mujeres y $52.2 \%$ hombres, cuyo rango de edad oscilaba entre los 19 y 69 años. La muestra de la fase 2 quedó conformada por 981 participantes donde el $62.39 \%$ fueron mujeres y $37.61 \%$ hombres, en esta ocasión las edades fueron de los 18 hasta los 69 años.

Los datos fueron obtenidos mediante dos modalidades: lápiz papel y digital (Formularios Google). Durante la prueba piloto aplicamos el instrumento en su versión lápiz papel. Para ello, acudimos a una empresa mexicana del sector alimenticio que aceptó participar y presentamos un oficio explicando el propósito del estudio y el aviso de confidencialidad. Tras obtener el consentimiento informado, procedimos a la aplicación del instrumento dentro de sus instalaciones.

A fin de agilizar la recolección de datos para la fase dos, optamos por la modalidad digital en las otras organizaciones participantes. Capturamos el instrumento en la herramienta "Formularios Google", generamos una liga y la compartimos por distintintos medios electrónicos (correo, redes sociales, etc). Se incluyó el aviso de confidencialidad al inicio del instrumento a fin de garantizarles a los participantes y sus organizaciones el anonimato. Ambos procedimientos de obtención de datos, ocurrieron a finales del 2019.

\section{Diseño de estudio}

El estudio fue ex post facto, cuantitativo no experimental de tipo transversal, ya que se observó el fenómeno (tecnoadicción) tal y como 
se da en el contexto natural y la recolección de datos se hizo en un solo momento (Appelbaum et al., 2018; Hernández Sampieri et al., 2014).

\section{Instrumento para la recolección de datos}

El instrumento que utilizamos en el presente estudio, fue la Escala de Tecnoadicción de Salanova et al. (2013) compuesta por seis reactivos con escala tipo Likert de siete puntos (donde 0 es nunca y 6 es siempre), que mide dos dimensiones, el uso compulsivo y excesivo de la tecnología. La estructura factorial presenta una consistencia interna (alfa de Cronbach) de $\alpha=0.85$ (ver Tabla 1 ).

\section{Resultados Fase 1}

\section{Adaptación cultural y evaluación interjueces}

La propuesta quedó conformada por 10 reactivos, de los cuales, 3 son de la escala original, 4 son propuestas de adaptación y 3 son nuevos reactivos (véanse Tabla 2 y 3 ).

\section{Tabla 2}

Tabla 2

Evaluación interjueces para la escala de tecnoadicción

\begin{tabular}{|c|c|c|c|c|c|c|c|c|}
\hline Factores & Reactivos & & $\begin{array}{c}\text { Juez } \\
1\end{array}$ & $\begin{array}{c}\text { Juez } \\
2\end{array}$ & $\begin{array}{c}\text { Juez } \\
3\end{array}$ & $\begin{array}{c}\text { Juez } \\
4\end{array}$ & $\begin{array}{c}\text { Juez } \\
5\end{array}$ & Decisión \\
\hline \multirow{3}{*}{$\begin{array}{l}\text { Uso } \\
\text { excesivo } \\
\text { de la } \\
\text { tecnología }\end{array}$} & $\begin{array}{c}\text { Creo que utilizo en } \\
\text { exceso las tecnologías } \\
\text { en mi vida. }\end{array}$ & Original & 1 & 1 & 1 & 1 & 1 & Conservar \\
\hline & $\begin{array}{l}\text { Utilizo continuamente } \\
\text { las tecnologías, incluso } \\
\text { fuera de mi horario de } \\
\text { trabajo. }\end{array}$ & Original & 1 & 1 & 1 & 1 & 1 & Conservar \\
\hline & $\begin{array}{c}\text { Me gusta pasar largas } \\
\text { horas usando } \\
\text { tecnologías. }\end{array}$ & Nuevo & 1 & 1 & 1 & 1 & 1 & Conservar \\
\hline \multirow{3}{*}{$\begin{array}{l}\text { Uso } \\
\text { compulsiv } \\
\text { o de la } \\
\text { tecnología }\end{array}$} & $\begin{array}{c}\text { Siento que un impulso } \\
\text { interno me obliga a } \\
\text { utilizar las tecnologías } \\
\text { en cualquier momento } \\
\text { y lugar. }\end{array}$ & Original & 1 & 1 & 1 & 1 & 1 & Conservar \\
\hline & $\begin{array}{c}\text { Siento una enorme } \\
\text { necesidad de utilizar la } \\
\text { tecnologías en } \\
\text { momentos que no son } \\
\text { adecuados (p. ej., al } \\
\text { manejar). }\end{array}$ & Nuevo & 1 & 1 & 1 & 1 & 1 & Conservar \\
\hline & $\begin{array}{l}\text { Me siento incómodo } \\
\text { cuando no puedo } \\
\text { utilizar tecnologías. }\end{array}$ & Nuevo & 1 & 1 & 1 & 1 & 1 & Conservar \\
\hline
\end{tabular}


Tabla 3

Tabla 3

Evaluación interjueces para la escala de tecnoadicción (reactivos del instrumento original adaptados)

\begin{tabular}{|c|c|c|c|c|c|c|c|c|}
\hline Factores & $\begin{array}{l}\text { Reactivos } \\
\text { originales }\end{array}$ & $\begin{array}{c}\text { Propuesta de } \\
\text { modificación }\end{array}$ & $\begin{array}{c}\text { Juez } \\
1 \\
\end{array}$ & $\begin{array}{c}\text { Juez } \\
2 \\
\end{array}$ & $\begin{array}{c}\text { Juez } \\
3 \\
\end{array}$ & $\begin{array}{c}\text { Juez } \\
4 \\
\end{array}$ & $\begin{array}{c}\text { Juez } \\
5\end{array}$ & Decisión \\
\hline \multirow{2}{*}{$\begin{array}{c}\text { Uso } \\
\text { excesivo de } \\
\text { la } \\
\text { tecnología }\end{array}$} & \multirow{2}{*}{$\begin{array}{lr}\text { Dedico } & \text { más } \\
\text { tiempo a las } \\
\text { tecnologías } \\
\text { a estar } \\
\text { amigos con } \\
\text { familiares o a } \\
\text { practicar } \\
\text { hobbies } \\
\text { actividades } \\
\text { tiempo libre. } \\
\end{array}$} & $\begin{array}{l}\text { Dedico más } \\
\text { tiempo a usar } \\
\text { las tecnologías } \\
\text { que a estar con } \\
\text { mis amigos y } \\
\text { familiares. }\end{array}$ & 1 & 1 & 1 & 1 & 1 & Conservar \\
\hline & & $\begin{array}{l}\text { Dedico más } \\
\text { tiempo a usar } \\
\text { las tecnologías } \\
\text { que ha practicar } \\
\text { algún deporte o } \\
\text { actividad al aire } \\
\text { libre. }\end{array}$ & 1 & 1 & 1 & 1 & 1 & Conservar \\
\hline \multirow[t]{2}{*}{$\begin{array}{c}\text { Uso } \\
\text { compulsivo } \\
\text { de la } \\
\text { tecnología }\end{array}$} & $\begin{array}{l}\text { Me encuentro } \\
\text { pensando en } \\
\text { tecnologias } \\
\text { continuamente } \\
\text { (por ejemplo, } \\
\text { revisar el correo } \\
\text { electrónico, } \\
\text { búsqueda de } \\
\text { información en } \\
\text { internet, etc.) } \\
\text { Incluso fuera } \\
\text { del horario de } \\
\text { trabajo. }\end{array}$ & $\begin{array}{l}\text { Me encuentro } \\
\text { pensando en } \\
\text { tecnologías } \\
\text { continuamente } \\
\text { (por ejemplo, en } \\
\text { revisar el correo } \\
\text { electrónico, } \\
\text { buscar } \\
\text { información en } \\
\text { internet, utilizar } \\
\text { redes sociales } \\
\text { etc.) Incluso } \\
\text { fuera del horario } \\
\text { de trabajo. }\end{array}$ & 1 & 1 & 1 & 1 & 1 & Conservar \\
\hline & $\begin{array}{l}\text { Me siento mal si } \\
\text { no tengo } \\
\text { acceso a las } \\
\text { tecnologías } \\
\text { (Internet, correo } \\
\text { electrónico, } \\
\text { móvil, etc.). }\end{array}$ & $\begin{array}{l}\text { Me siento mal si } \\
\text { no tengo } \\
\text { acceso a las } \\
\text { tecnologias } \\
\text { (Internet, correo } \\
\text { electrónico, } \\
\text { teléfono celular, } \\
\text { etc.). }\end{array}$ & 1 & 1 & 1 & 1 & 1 & Conservar \\
\hline
\end{tabular}

Test KMO y Bartlett

Los resultados obtenidos en KMO (puntaje 0.869) y en el Test de esfericidad de Bartlett (puntajes menores a 0.005 ), indicaron que era apropiado aplicar el análisis factorial (ver Tabla 4).

Tabla 4

\section{Tabla 4}

Prueba KMO y Bartlett para la escala de tecnoadicción

$\begin{array}{lc}\text { KMO } & 0.869 \\ \text { Bartlett } & 575.176\end{array}$

GI

$P$ 


\section{Análisis factorial exploratorio}

La Tabla 5 nos muestra la agrupación de los reactivos de la escala de tecnoadicción, la cual quedó conformada por dos factores que explican el $60.79 \%$ de la varianza. El factor, 'uso compulsivo de la tecnología' quedó compuesto por 5 reactivos que explican el $34.76 \%$ de la varianza. El factor uso excesivo de la tecnología quedó con tres reactivos que explican el $26.03 \%$ de la varianza. La estructura factorial resultante del análisis concuerda con las estructuras factoriales presentadas por Llorens et al. (2011). Durante este análisis se perdieron dos reactivos, cuyos coeficientes eran menores a 0.4 .

\section{Tabla 5}

Tabla 5

\begin{tabular}{|c|c|c|c|}
\hline \multicolumn{2}{|c|}{ Escala de techoadiccion (subescalas, cargas tactoriales e ind } & \multicolumn{2}{|c|}{ Subescalas } \\
\hline \multicolumn{2}{|r|}{ Reactivos } & $\begin{array}{l}\text { Uso } \\
\text { compulsivo }\end{array}$ & $\begin{array}{c}\text { Uso } \\
\text { excesivo }\end{array}$ \\
\hline \multirow{8}{*}{$\begin{array}{l}\text { Cargas } \\
\text { factoriales }\end{array}$} & $\begin{array}{l}\text { 8. Me siento mal si no tengo acceso a las } \\
\text { tecnologías (internet, correo electrónico, teléfono } \\
\text { celular, etc.). }\end{array}$ & 0.799 & \\
\hline & $\begin{array}{l}\text { 6. Siento que un impulso interno me obliga a } \\
\text { utilizar las tecnologias en cualquier momento y } \\
\text { lugar. }\end{array}$ & 0.767 & \\
\hline & $\begin{array}{l}\text { 2. Siento una enorme necesidad de utilizar las } \\
\text { tecnologías en momentos que no son adecuados } \\
\text { (p.ej., al manejar). }\end{array}$ & 0.694 & \\
\hline & $\begin{array}{l}\text { 4. Me encuentro pensando en tecnologias } \\
\text { continuamente (p. ej. en revisar el correo } \\
\text { electrónico, buscar información en internet, utilizar } \\
\text { redes sociales, etc.) incluso fuera del horario de } \\
\text { trabajo. }\end{array}$ & 0.638 & \\
\hline & $\begin{array}{l}\text { 7. Me siento incómodo cuando no puedo utilizar } \\
\text { tecnologías. }\end{array}$ & 0.633 & \\
\hline & $\begin{array}{l}\text { 5. Dedico más tiempo a usar las tecnologías que a } \\
\text { practicar algún deporte o actividad al aire libre. }\end{array}$ & & 0.849 \\
\hline & $\begin{array}{l}\text { 1. Dedico más tiempo a usar las tecnologías que a } \\
\text { estar con mis amigos y familiares. }\end{array}$ & & 0.800 \\
\hline & $\begin{array}{l}\text { 3. Me gusta pasar largas horas usando } \\
\text { tecnologías. }\end{array}$ & & 0.642 \\
\hline \multicolumn{2}{|c|}{ Número de reactivos } & & \\
\hline & & & 3 \\
\hline $\begin{array}{l}\text { \% de varianz } \\
\alpha \text { de Cronba }\end{array}$ & explicada & $34.761 \%$ & $26.028 \%$ \\
\hline \multicolumn{4}{|c|}{ Correlaciones interfactor } \\
\hline \multirow{2}{*}{\multicolumn{2}{|c|}{$\begin{array}{l}\text { Uso compulsivo de la tecnología } \\
\text { Uso excesivo de la tecnología }\end{array}$}} & 1 & \\
\hline & & $0.592^{*}$ & 1 \\
\hline \multicolumn{2}{|c|}{ Media (media teórica $=3.5$ ) } & 2.22 & 2.25 \\
\hline \multirow{2}{*}{\multicolumn{2}{|c|}{ Desviación estándar }} & & \\
\hline & & 1.500 & 1.599 \\
\hline
\end{tabular}

\section{Resultados fase 2}

\section{Análisis factorial confirmatorio mediante ecuaciones estructurales}

Al poner a prueba la estructura factorial resultante del análisis factorial exploratorio (varimax), mediante ecuaciones estructurales, confirmamos el modelo de dos factores propuesto por Llorens et al. (2011) (ver Figura 
1). Asimismo, los índices de bondad de ajuste cumplieron con los criterios estipulados en el procedimiento: el puntaje de Chi-cuadrada (CMIN) no fue significativo, los puntajes de CFI, TLI y NFI son mayores a 0.95 , RMSEA es menor a 0.08 y las cargas factoriales en el modelo teórico fueron superiores a 0.07 (véase Tabla 6).

Tabla 6

\section{Tabla 6}

\section{Estadisticos de bondad de ajuste de la escala de tecnoadicción}

Chi-cuadrada (CMIN).

GI

$P$

CMIN/DF

$\mathrm{NFI}$

$\mathrm{RFI}$

IFI

TLI

$\mathrm{CFI}$

RMSEA
100.580

19

0.000

5.294

0.965

0.949

0.972

0.958

0.971

0.066 
Figura 1

Modelo de estructura factorial de la escala de tecnoadicción (elaboración propia)

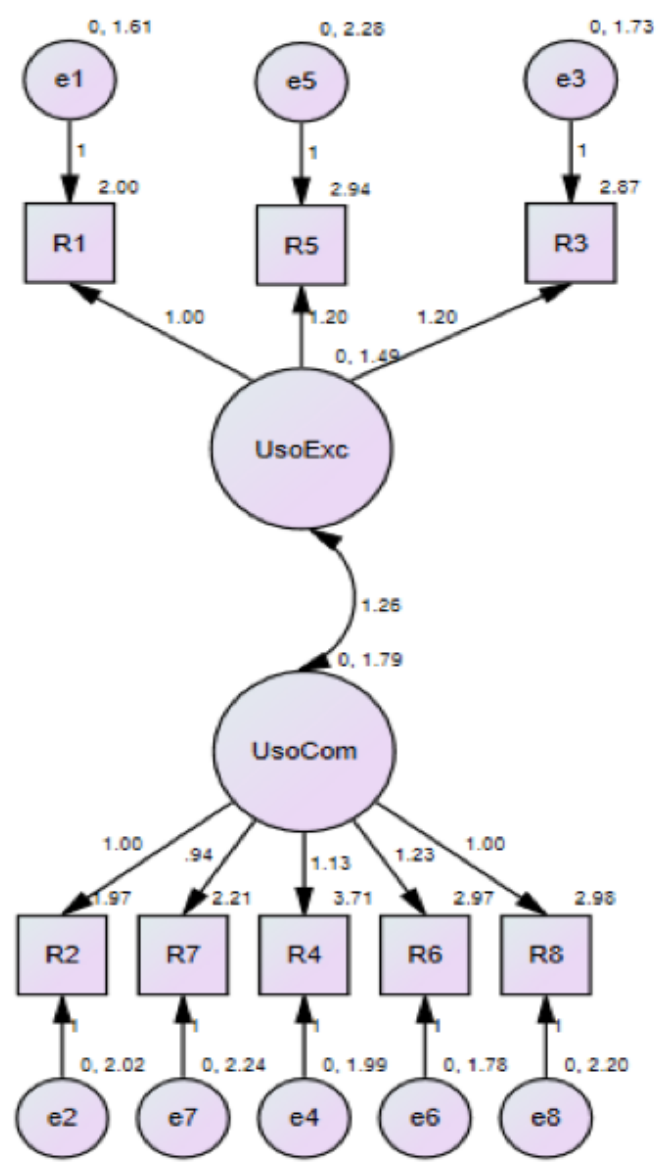

Figura 1

Análisis de confrabilidad

Los índices de consistencia interna (alfa de Cronbach) de cada factor en la escala final obtuvieron puntajes por encima del criterio 0.70 (ver Tabla 5).

\section{Discusión}

Los resultados obtenidos tras el proceso de adaptación y validación, permitieron identificar que la escala de tecnoadicción que proponemos, es útil para medir la dependencia tecnológica, al cumplir con los criterios psicométricos necesarios para aplicarse en población laboral mexicana.

A través de la evaluación interjueces logramos ratificar la validez de contenido del instrumento. Los expertos corroboraron que los reactivos propuestos, concordaban con la literatura revisada y que la redacción era lo suficientemente clara para ser entendida por la población mexicana (Hernández Sampieri et al., 2014; Reyes \& Barragán, 2008). Durante el 
análisis factorial exploratorio, únicamente se perdieron dos reactivos de la escala original ("Creo que utilizo en exceso las tecnologías en mi vida" y "Utilizo continuamente las tecnologías, incluso fuera de mi horario de trabajo") ya que sus coeficientes no fueron mayores o iguales a 0.4 (Hernández Sampieri et al., 2014).

Como puede observarse en la Figura 1, a través de la técnica de ecuaciones estructurales, fueron confirmados dos factores, 'el uso excesivo de la tecnología', compuesto por tres reactivos, dos que fueron sometidos a modificación [R1. Dedico más tiempo a usar tecnologías que a estar con mis amigos y familiares; y R5. Dedico más tiempo a usar las tecnologías que a practicar algún deporte o actividad al aire libre] y uno nuevo [R3. Me gusta pasar largas horas usando tecnologías]. Y 'el uso compulsivo de la tecnología', compuesto por cinco reactivos, dos que fueron sometidos a modificación [R4. Me encuentro pensando en tecnología continuamente (p.ej. en revisar el correo electrónico, buscar información en internet, utilizar redes sociales, etc.) Incluso fuera de mi horario de trabajo; y R8. Me siento mal si no tengo acceso a las tecnologías (internet, correo electrónico, teléfono celular, etc.)], uno de la escala original [R6. Siento que un impulso interno me obliga a utilizar las tecnologías en cualquier momento y lugar] y dos nuevos [R2. Siento una enorme necesidad de utilizar las tecnologías en momento que no son adecuados (p. ej. al manejar); y R7. Me siento incómodo cuando no puedo utilizar tecnologías].

El instrumento final quedó compuesto por ocho reactivos que obtuvieron cargas factoriales significativas (mayores a 0.07 ) y que lograron adaptarse al modelo diseñado en AMOS cuyos índices de bondad de ajuste son aceptables (Chi-cuadrada $=100.580 ; \mathrm{Gl}=19$; CMIN/ $\mathrm{DF}=5.294 ; \mathrm{p}=.000 ; \mathrm{NFI}=.965 ; \mathrm{TLI}=.958 ; \mathrm{CFI}=.971 ; \mathrm{yMSEA}=.066$ ) (Ruiz et al., 2010). Lo anterior permite constatar la congruencia conceptual que las dimensiones resultantes de los análisis factoriales (mediante varimax y ecuaciones estructurales), mantienen con el modelo teórico planteado por Llorens et al. (2011).

Por último, en el análisis de consistencia interna, los puntajes alfa de Cronbach que obtuvieron de los factores, confirmaron la confiabilidad de la escala, ya que superaron el criterio 0.70 (Hernández Sampieri et al., 2014), (uso excesivo de la tecnología $\alpha=0.749$; uso compulsivo de la tecnología $\alpha=0.801$; y total de la escala $\alpha=0.845$ ) (ver Tabla 5). Estos resultados presentan similitudes con lo reportado por Llorens et al. (2011).

A diferencia de otros estudios psicométricos sobre tecnoadicción en México, en donde únicamente se presenta el análisis factorial exploratorio, la presente investigación cuenta con un análisis confirmatorio mediante ecuaciones estructurales en una muestra más robusta. Asimismo, ofrece una nueva herramienta para futuros estudios, ya que de acuerdo con la literatura revisada, la mayoría de los investigadores utilizan la versión al español del IAT. Además de estas ventajas, también tiene ciertas limitaciones: Una de ellas es que no mide fatiga y ansiedad derivada de la tecnoadicción. Decidimos no utilizar 
los reactivos de ansiedad y fatiga que están presentes en el cuestionario RED-Tecnoestrés (Salanova et al., 2013) ya que están configurados para medir los fenómenos como consecuencia del estrés derivado de las TIC (por ejemplo, me siento tenso y ansioso cuando trabajo con tecnologías. después de usar tecnologías me cuesta trabajo prestar atención a otras actividades). Para tratar esta limitación, en próximos estudios se podrían reformular los reactivos y analizar sus propiedades psicométricas (como lo hicimos aquí) o hacer un estudio correlacional entre la tecnoadicción y las variables ansiedad y fatiga (apoyándose con instrumentos estandarizados que midan estos fenómenos). El contar con reactivos que miden la adicción a las TICS en términos muy generales puede ser una limitante, es por ello que consideramos que para los próximos estudios se incluya algún reactivo en donde el usuario especifique qué tecnología es la que más utiliza y por qué lo hace. Lo anterior permitirá identificar las propiedades reforzadoras que tienen los dispositivos tecnológicos en la conducta de los usuarios mexicanos.

\section{Conclusión}

A lo largo del tiempo, la conceptualización del término adicción, ha girado en torno al consumo de sustancias. Sin embargo, se sabe que algunos hábitos conductuales pueden aparentar ser inofensivos, pero en determinadas circunstancias, suelen convertirse en trastornos adictivos que gradualmente interfieren de manera grave, en la vida de las personas (Cía, 2013); tal es el caso de la tecnoadicción. Pese a las evidencias, aún queda la duda de si verdaderamente nos encontramos ante una adicción conductual, o a un uso normal (Terán Prieto, 2019). El aumento en la disponibilidad ambiental de TIC es un beneficio para la humanidad. No obstante, la falta de recursos personales, como el pobre control cognitivo (Gil, 2015), o algunos rasgos de la personalidad de los usuarios (timidez excesiva, introversión, inseguridad, baja autoestima, rechazo de la imagen corporal, falta de identidad, etcétera) pueden favorecer la aparición del fenómeno (Terán Prieto, 2019).

Con los hallazgos de la presente investigación, aportamos una escala para la cultura laboral mexicana. Herramienta psicométrica que posibilitará el estudio de la tecnoadicción y que permitirá seguir entendiendo su etiología, estudiar la relación que mantiene con otros factores de riesgo psicosocial y diseñar programas de prevención e intervención a fin de mejorar la relación entre los usuarios y las TIC, creando a su vez espacios de trabajo saludables. Así mismo, fortalecemos el entendimiento de las denominadas adicciones conductuales para su posible reconocimiento como enfermedades en un futuro próximo, en el DSM-V.

Este tipo de estudios contribuye a ofrecer herramientas de medición confiable, válida y estandarizada para la población en cuestión, que permita realizar más estudios que arrojen evidencia de las características de esta variable en el país, considerado como uno de los que más usan la Internet en América Latina (INEGI, Secretaría de Comunicaciones 
y Transportes, \& IFT, 2020). Esto implicará que las autoridades correspondientes tengan información disponible y actualizada para realizar campañas de prevención y sensibilización a la población objetivo.

\section{Referencias}

American Psychiatric Association. (2014). Manual diagnóstico y estadístico de los trastornos mentales (DSM-5) ( $5^{\mathrm{a}} \mathrm{ed}$.). Editorial Médica Panamericana.

Appelbaum, M., Cooper, H., Kline, R. B., Mayo-Wilson, E., Nezu, A. M., \& Rao, S. M. (2018). Journal article reporting standards for quantitative research in psychology: The APA Publications and Communications Board task force report. American Psychologist, 73(1), 3-25. http://dx.doi.org/10.10 37/amp0000191

Asociación de Internet MX. (2019). $15^{\circ}$ Estudio sobre los hábitos de los usuarios de internet en México. https://irp-cdn.multiscreensite.com/81280eda/file s/uploaded/15\%2BEstudio\%2Bsobre\%2Blos\%2BHa_bitos\%2Bde\%2Bl os\%2BUsuarios\%2Bde\%2BInternet\%2Ben\%2BMe_xico\%2B2019\%2Bv ersio_n\%2Bpu_blica.pdf

Aznar-Díaz, I., Romero-Rodríguez, J. M., García-González, A., \& RamírezMontoya, M. S. (2020). Mexican and Spanish university students' internet addiction and academic procrastination: Correlation and potential factors. PloS one, 15(5), e0233655. https://doi.org/10.1371/journal.pon e.0233655

Bernal Nava, N. (2011). Estudio de la adicción a internet en los jóvenes en la actualidad en México [Tesis de licenciatura]. Universidad Autónoma del Estado de México, México.

Cabero-Almenara, J., Pérez-Díez de los Ríos, J. L., \& Valencia-Ortiz, R. (2020). Escala para medir la adicción de estudiantes a las redes sociales. Convergencia, 27. https://doi.org/10.29101/crcs.v27i83.11834

Chahín, N., \& Libia, B. (2016). Propiedades psicométricas del Cuestionario de Adicción a Internet y a los Videojuegos para Adolescentes. Universitas Psychologica, 17(4), 1-13. https://doi.org/10.11144/Javeriana.upsy17-4.p pca

Chen, K., Tarn, J. M., \& Han, B. T. (2004). Internet dependency: Its impact on online behavioral patterns in E - commerce. Human Systems Management, 23(1), 49-58.

Cía, A. H. (2013). Las adicciones no relacionadas a sustancias (DSM-5, APA, 2013): un primer paso hacia la inclusión de las adicciones conductuales en las clasificaciones categoriales vigentes. Revista de Neuro-Psiquiatría, 76(4), 210-217. https://doi.org/10.20453/rnp.v76i4.1169

Contreras, J. H., Martínez, M. O., Almaguer, J. M., Ramírez, A., \& Miramontes, A. (2019). Adiccion a internet: el caso de adolescentes de cinco escuelas secundarias de mexico. Enseñanza e Investigación en Psicologia, (SI), 34-46.

Escobedo Portillo, M. T., Hernández Gómez, J. A., Estebané Ortega, V., \& Martínez Moreno, G. (2016). Modelos de ecuaciones estructurales: características, fases, construcción, aplicación y resultados. Ciencia \& Trabajo, 18(55), 16-22. http://dx.doi.org/10.4067/S0718-24492016000 100004 
Garcia-Priego, B. A., Triana-Romero, A., Pinto-Galvez, S. M., Duran-Ramos, C., Salas-Nolasco, O., Reyes, M. M., ... \& Troche, J. M. R. (2020). Anxiety, depression, attitudes, and internet addiction during the initial phase of the 2019 coronavirus disease (COVID-19) epidemic: A cross-sectional study in Mexico. medRxiv. https://doi.org/10.1101/2020.05.10.20095844

Gentile, D. A., Choo, H., Liau, A., Sim, T., Li, D., Fung, D., \& Khoo, A. (2011). Pathological video game use among youths: A two-year longitudinal study. Pediatrics, 127(2), e319-e329. https://doi.org/10.1542/peds.2010-1353

Gil, A. (2015). Tecnoadicción: una revisión teórica desde la psicología [Tesis, Universitat Jaume I]. Repositori UJI. http://repositori.uji.es/xmlui/bitst ream/handle/10234/137398/TFG_2014_gilA.pdf?sequence $=1$

Hernández Sampieri, R., Fernández Collado, C., \& Baptista Lucio, M. D. P. (2014). Metodología de la investigación. McGraw-Hill.

Hilt, J. A., de Korniejczuk, R. B., \& Collins, E. (2015). Adicción a internet en estudiantes universitarios mexicanos. Revista de Investigación Universitaria, 4(2), 11-17. https://doi.org/10.17162/riu.v4i2.681

Instituto Nacional de Estadística y Geografía (INEGI), Secretaría de Comunicaciones, \& Transportes e Instituto Federal de Telecomunicaciones (IFT). (2020).Encuesta nacional sobre disponibilidad $y$ uso de tecnologías de la información en los hogares, 2019. https://www.inegi.org.mx/contenidos/saladeprensa/boletines/20 20/OtrTemEcon/ENDUTIH_2019.pdf

Jasso-Medrano, J. L., \& Lopez-Rosales, F. (2018). Measuring the relationship between social media use and addictive behavior and depression and suicide ideation among university students. Computers in Human Behavior, 87, 183-191. https://doi.org/10.1016/j.chb.2018.05.003

Kim, Y., Park, J. Y., Kim, S. B., Jung, I. K., Lim, Y. S., \& Kim, J. H. (2010). The effects of Internet addiction on the lifestyle and dietary behavior of Korean adolescents. Nutrition Research and Practice, 4(1), 51-57. https://doi.org /10.4162/nrp.2010.4.1.51

Kircaburun, K., \& Griffiths, M.D. (2018). The dark side of internet: Preliminary evidence for the associations of dark personality traits with specific online activities and problematic internet use. Journal of Behavioral Addictions, 7(4), 993-1003. https://doi.org/10.1556/2006.7.2018.109

Li, C., Dang, J., Zhang, Q., \& Guo, J. (2014). Internet addiction among Chinese adolescents: The effect of parental behavior and self-control. Computers in Human Behavior, 41, 1-7. https://doi.org/10.1016/j.chb.2014.09.001

Lin, Y. H., Chang, L. R., Lee, Y. H., Tseng, H. W., Kuo, T. B., \& Chen, S. H. (2014). Development and validation of the Smartphone Addiction Inventory (SPAI). PloS one, 9(6), e98312. https://doi.org/10.1371/jour nal.pone.0098312

Llorens, S., Salanova, M., \& Ventura, M. (2011). Guias de intervención. Tecnoestrés. Editorial Síntesis.

Lloret, D., Morell, R., Marzo, J., \& Tirado, S. (2017). Validación española de la Escala de Adicción a Videojuegos para Adolescentes (GASA). Atención Primaria, 50(6), 350-358. https://doi.org/10.1016/j.aprim.2017.03.015

McEwen, B. S. (2006). Protective and damaging effects of stress mediators: Central role of the brain. Dialogues in Clinical Neuroscience, 8(4), 367-381. 
Montoya, O. (2007). Aplicación del análisis factorial a la investigación de mercados. Caso de estudio. Scientia et Technica, 1(35), 281-285. https:// doi.org/10.22517/23447214.5443

Organización para la Cooperación y el Desarrollo Económicos (OCDE). (2017). Estudios sobre telecomunicaciones y radiodifusión en México 2017. http://www.ift.org.mx/sites/default/files/estudio_de_la_ocde_so bre_telecomuncaciones_y_radiodifusion_en_mexico_2017.pdf

Reyes, L., \& Barragán, I. (2008). Procedimiento de validación psicométrica culturalmente relevante. En La psicología social en México, XII. AMPESO.

Riedl, R. (2012). On the biology of technostress: Literature review and research agenda. ACM SIGMIS Database: The database for advances in information systems, 44(1), 18-55. https://doi.org/10.1145/2436239.2436242

Ruiz-Palmero, J., Sánchez-Rivas, E., Gómez-García, M., \& Sánchez Vega, E. (2019). Future teachers' smartphone uses and dependence. Education Sciences, 9(3), 194. https://doi.org/10.3390/educsci9030194

Ruiz, M. A., Pardo, A., \& San Martín, R. (2010). Modelos de ecuaciones estructurales. Papeles del Psicólogo, 31(1), 34-45.

Salanova, M., Llorens, S., \& Cifre, E. (2013). The dark side of technologies: Technostress among users of information and communication technologies. International Journal of Psychology, 48(3), 422-436. https:/ /doi.org/10.1080/00207594.2012.680460

Sánchez-Carbonell, X. (2014). Adicciones tecnológicas: ¿quéson y cómo tratarlas? Editorial Síntesis.

Sánchez-Carbonell, X., Beranuy, M., Castellana, M., Chamarro, A., \& Oberst, U. (2008). La adicción a Internet y al móvil: ¿moda otra trastorno? Adicciones, 20(2), 149-159.

Shapira, N. A., Lessig, M. C., Goldsmith, T. D., Szabo, S. T., Lazoritz, M., Gold, M. S., \& Stein, D. J. (2003). Problematic internet use: Proposed classification and diagnostic criteria. Depression and Anxiety, 17(4), 207-216. https://doi.org/10.1002/da.10094

Simó Sanz, C., Martínez Sabater, A., Ballestar Tarín, M. L., \& Domínguez Romero, A. (2017). Instrumentos de evaluación del uso problemático del teléfono móvil/smartphone. Saludy Drogas, 17(1), 5-14.

Terán Prieto, A. (2019). Ciberadicciones. Adicción a las nuevas tecnologías (NTIC). En A. E. Pap (Ed.), Congreso de actualización pediatría 2019 (pp. 131-141). Lúa Ediciones. https://www.aepap.org/sites/default/files/pags ._131-142_ciberadicciones.pdf

Tsitsika, A., Tzavela, E., Mavromati, F., \& EU NET ADB Consortium. (2013). Investigación sobre conductas adictivas a internet entre los adolescents europeos. European Union. http://www.injuve.es/sites/default/files/201 3/03/publicaciones/FinalResearchInternet-ES.pdf

Vink, J. M., Van Beijsterveldt, T. C., Huppertz, C., Bartels, M., \& Boomsma, D. I. (2016). Heritability of compulsive internet use in adolescents. Addiction Biology, 21(2), 460-468. https://doi.org/10.1111/adb.12218 